\title{
THE ANTENNA SOFTWARE INITIATIVE (ASI) WITHIN ACE: RESULTS AND FUTURE
}

\author{
G. A. E. Vandenbosch* and Raphaël Gillard ${ }^{\dagger}$ \\ * Katholieke Universiteit Leuven, ESAT-TELEMIC, \\ B-3001 Leuven, Belgium \\ guy.vandenbosch@esat.kuleuven.be; \\ ${ }^{\dagger} \mathrm{IETR}$, INSA, \\ 35043 Rennes, France \\ raphael.gillard@insa-rennes.fr
}

\begin{abstract}
In this paper the results of the four years of work within the Antenna Software Initiative (ASI) of ACE will be analyzed. Also, a view will be given on a possible future for this European cooperation framework. The reasons for the ASI have been explained in the special ASI session at EuCAP 2006 [1]. The primary goal of the ASI was to build a European framework that allows research groups active in the development of antenna analysis and design software to intensify and optimize cooperative research.
\end{abstract}

Two high level activities have been performed:

1. benchmarking of existing software codes, and

2. laying the foundations for a European software library.

The benchmarking activity was organized because, right from the beginning, a clear lack of thoroughly investigated, welldescribed benchmarking calculations was felt by all participants in the software activity.

The European software library is a long term project, aiming at building a library of software routines performing generic functions that are widely used in the field of and typical for antenna and more general electromagnetic modeling. Within the ASI, the first problem encountered, the problem of interfacing has been tackled by the development of the Electromagnetic Data Interface (EDI). The work consisted of defining a standardized way of storing electromagnetic data (currents, fields, ...) in files (the so-called Data Dictionaries), and of developing the necessary software routines to do this automatically through a simple routine call. The status of this interface and the procedure that researchers have to follow to make use of it, will be explained.

The power of a future software library has been proven through numerous integration projects, where several ASI partners joined forces. They have shown that by making use of each others routines, complementary competences can lead to remarkable results, results that could never be reached by a single partner. This activity also provided the partners involved of a better understanding of the problems encountered during integration of actual software, giving them the necessary insight for future cooperation.

It has to be emphasized that the ASI structure is a structure set up in a democratic way to cope with the most important bottle necks encountered towards European antenna software cooperation. It was funded by the ACE Network of Excellence. One of the main challenges to be coped with is the future funding of this type of cooperation. Also this issue will be addressed in the paper.

\section{Result 1: Inventory}

within the ACE network in a first step an inventory was made of the software available among all partners of ACE, describing in detail the possibilities and limitations (capabilities to handle complex structures, environments, feeds, active components,...). This was the basis for all further work within the ASI. The result of this inventory can be consulted at the ACE website [2].

\section{Result 2: Benchmarking structuring}

In ACE-1 (2004-2005), benchmarking of antenna software has been organized. A special online service, called SoftLAB (Software on Line Antenna Benchmark) has been developed within VCE, the ACE portal. SoftLAB enables anyone to:

- Download description files for the proposed testcases.

- Participate in the benchmarking process by uploading simulated results for one or several of the proposed test-cases.

- Have an overview of the obtained results (for completed benchmark runs).

SoftLAB is open to anyone developing or using antenna software tools. The only condition to access SoftLAB is to register in the ACE community.

A first benchmark run (run \#1) has also been organized in 2004. Less than one year after the benchmark has been opened, 36 simulations had already been realized. 14 different participants took part to this benchmark and 18 different software tools (11 in-house and 7 commercial) had been used. 
In 2006, within ACE-2, a new benchmark run (run \#2) has been launched. The main originality of this new run was to focus on finite antenna arrays whose modelling is particularly challenging for simulation tools. The benchmark was also a blind process and no results were delivered before the complete closure of Run \#2 in December 2006. The benchmark was largely open as anyone belonging to the $\mathrm{ACE}$ community could participate in the simulation process thanks to SoftLAB. Several dissemination actions were organized to foster external participation (mailing, leaflets and meetings). Connections have been established with other activities (A1.2 and A2.4) in the selection process of test-cases in order to account for the industrial demand.

The main results of Run\#2 are the followings:

- 11 challenging structures have been proposed and discussed as possible benchmark test-cases (following a call for proposals within $\mathrm{ACE}$ participants)

- 7 structures have been selected for an open benchmark run (this run is open to everyone thanks to SoftLAB) - 4 of them were proposed in coordination with other ACE-activities

- 1 structure has been selected for a private benchmark run (this run is only open to participants in the benchmark WP for confidentiality purposes)

- 14 different laboratories and/or companies have contributed by simulating one or more test-cases; 2 participants are not members in $\mathrm{ACE}-2$ but extemal software vendors

- 28 simulations have been achieved

- 17 different antenna software tools have been assessed.

The simulation process for Run\#2 was stopped on the $6^{\text {th }}$ of December 2006 and simulated results were published in SoftLAB together with measured results. However, the simulation process is still continuing in 2007 for 2 structures (out of the 8 selected test-cases). This will give additional time to anyone wishing to simulate these very challenging test-cases (reflectarray antennas).

A new benchmark run has been organized in 2007 (Run\#3). The idea is to extend the scope of the benchmark by including the environment of the antenna in the simulation process. For instance, the simulation of a mobile antenna close to a human head is considered. Other test-cases involving MEMS elements are also addressed in order to assess the capabilities of antenna software to simulate non antenna problems.

All the results of the benchmark can be found in the VCE using the SoftLAB menu.
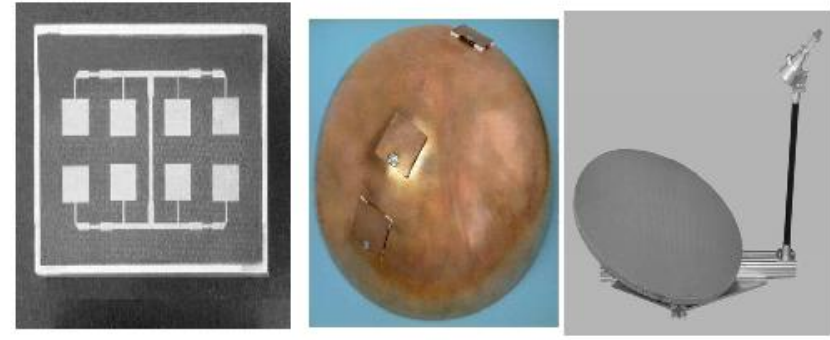
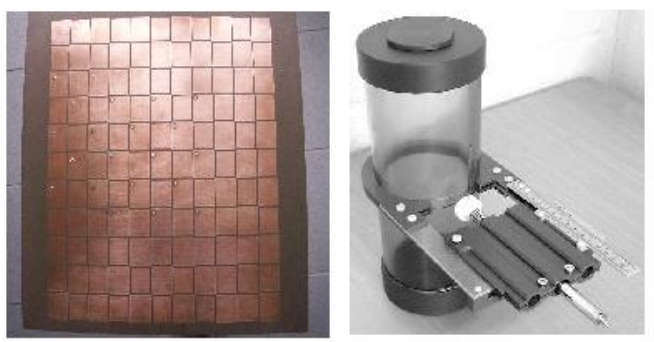

Fig. 1. These pictures illustrate some of the test-cases that have been selected for the ACE benchmark process (run \#2). The first one is a spherical conformal array (proposed by University of Zagreb), the second is a large reflectarray (proposed by University of Madrid), the third is a dipole with lossy cylinder that can be used for dosimetry tests (proposed by Chalmers), the fourth is a printed array with tight mutual coupling (proposed by LEAT, Nice) and the fifth is a small array with waveguide feeding. All test-cases are presented in details in SoftLAB.

\section{Contributors}

IDS, KUL, FT R\&D, TAS, CNRS-LEAT, IETR, IMST, POLITO, UNIFI, UNISI, UPC, UPM, UPV, CHALMERS, FOI, EPFL, UOB, UNIZAG, GET, SAPIENZA

\section{Result 3: File formats}

The work performed within the inventory phase pointed out that, in order to establish a lasting cooperation between partners cooperating in the construction of new software tools, a standardized file formatting is crucial. At this moment, many codes use their own formats and implementing communication between codes sometimes can be a real nightmare. Therefore, within the ASI, the idea of standardized file formats was pushed.

The definition of common file formats is more complex than it seems. The field covers a wide range of antenna types, modeled with completely different software tools. One of the key problems is that the data format of a specific software code will in most cases be influenced by the underlying method for solving a specific problem and often also by the software architecture.

The procedure that was followed within the ASI consisted of different steps.

First, the file types to be covered by the format must be determined. In a plenary meeting the ASI partners decided to prepare specifications for data files that in view of larger scale cooperation most frequently will be exchanged between antenna and electromagnetic software tools:

1. files with Green's functions as needed in tools using the Method of Moments,

2. files with electric (and magnetic) currents defined on meshes,

3. files containing near fields, including Time Domain fields on rectangular surfaces,

4. files with descriptions of modes (for example as in a waveguide) 
5. files with S-matrices,

6. files with far fields.

For these 6 quantities, so-called data dictionaries have been established to determine the exact contents of each file type. More precisely the data dictionary describes the physical entities to be stored in the file together with control data that explains how these physical data shall be interpreted.

- Near field data may e.g. be accompanied by specifications of a sampling grid and a coordinate system telling how the field sampling is positioned relative to some extemal coordinate system.

- Current data are linked to the description of a mesh, which is taken into consideration.

- $\quad \ldots$

The organization of the data in the file is very important. Questions like: 'What are the basic formats used and how and where do the different data blocks appear?' need to be answered. It was decided to base the new file format initially on the ASCI format such that files can be inspected.

It is important to mention that the data dictionaries are in the public domain and can be downloaded from the VCE.

\section{Result 4: Electromagnetic Data Interface}

Once the file formats have been defined, in principle the software codes (of the ASI partners) can be modified in order to make easy data exchange possible. However, ASI goes one step further. Imagine that the access to the files is made using a software library that stores and retrieves data in a controlled and uniform manner. This would reduce the modification effort en ormously, since it drastically reduces the chance for errors. The development of such library is as comprehensive as the specification of the format requirements themselves, but in our view it is worth the effort. One of the main requirements is that in practice, the use of the planned interface library, which has been denoted Electromagnetic Data Interface (EDI), should be very simple. The call of a library routine should be similar to the call of a simple read or write command for example in Fortran.

During the last three years, such a set of routines has been developed within the framework of $\mathrm{ACE}$, in cooperation with the European Space Agency. For three of the six quantities, near fields, far fields, and currents, a basic version of the EDI has been fully defined, implemented, and tested. All necessary developments have been done to use these routines in combination with fortran, C, and matlab codes. Several partners have already implemented the EDI in their codes (TICRA, THALES, SATIMO, KUL, ...). At KUL, the EDI is already being used in a project to exchange data within the framework of a project on the modeling of conducting textiles.

Here too, it is important to mention that at the end of $\mathrm{ACE}$, the EDI will be put in the public domain.

\section{Contributors}

IDS, KUL, TICRA, FT R\&D, TAS, CNRS-LEAT, IETR, SAPIENZA, POLITO, UNIFI, UNISI, UPC, UPM, UPV, FOI, EPFL, UOB, SATMO

\section{Result 5: Integration of software}

The last result is a very strategic one. Boosting the capabilities of software obviously can be done through developing new modelling techniques. However, this is fundamental research, not restructuring, and thus not the task of a NoE. It can also be done through integration of existing soffware. What is meant by this? In our view restructuring of research on antenna software does not mean trying to develop the "ultimate" monolithic software package, a single tool that covers as many aspects as possible in antenna design. Instead, the brainstorming process within ACE has worked out a very specific view on integration, based on cooperation and combining complementary software capabilities. Within $\mathrm{ACE}$, numerous integration activities have been perforned. Many $\mathrm{ACE}$ related modelling/software papers at many conferences describe the results of this type of cooperation. In the session where this paper will be presented, a number of integration activities is described, for example the integration of the CBLU fast solver for very large systems of equations developed at UPC AntennaLab into the antenna simulation codes of the ASI partners UPM-UNEX, KUL, UPV and EPFL. The integration activities illustrate the ultimate goal of the software activity: a European cooperation in the field of the development of antenna analysis and design software. During the presentation of this paper, several other illustrations will be given of what can be reached in this way, and at which "cost".

\section{Contributors}

KUL, TAS, SAPIENZA, POLITO, UNIFI, UNISI, UPC, UPM, UPV, EPFL

\section{Future}

At the end of 2007 , the ACE financing will end. In the period after that ACE will face its biggest challenge, also for the software activity: how to organize the survival of all results accomplished. Although we do not have a crystal ball, a few things can be said with confidence:

- for the benchmarking:

SoftLAB is there and is being finalized so that it can easily be used after ACE. As long as the VCE (the Virtual Center of Excellence, at this moment ACE's website), is up and running we are confident about its survival.

EuCAP is there and can be used as a forum to organize further benchmarking activities in the future. The idea is to organize an open meeting each year at EuCAP where new benchmarking challenges are being discussed and defined. SoftLAB can then be used to handle this. 
The budget needed to continue the benchmarking activity in the future is certainly feasible.

- for the EDI:

The very basic routines have been developed and are available. They are used already by some partners. Since the further development of the EDI requires considerable budgets, the key issue here is whether a proper framework can be defined, with sufficient resources to continue its implementation and maintenance. This problem is not solved at this moment, but initiatives are taken. They involve EurAAP, the European Association on Antennas and Propagation, and ESA-ESTEC, the European Space Agency, which is strongly supporting this initiative.

- for the integration:

ACE-ASI was the first initiative in Europe propagating antenna software integration as one of its prime goals. In our personal view, and this has been confirmed in the field during the last four years, this activity lies very close to the natural interest of the researchers involved: to produce new scientific results that can be published in papers. We believe that the "little" change in mentality that ASI has invoked has a very good chance of surviving within the framework of a larger organization, for example EurAAP. It is only fair to note that these "integrations" do not absolutely need the EDI. The EDI only makes them much more efficient and less time consuming. The question in our view is thus not whether there will be integration in the future, but whether the EDI will be used for it.

\section{Conclusion}

ACE-ASI has worked for four years towards a very specific goal: the restructuring of the European scene on the development of antenna analysis and design software. The final goal is to work out and implement a model of cooperation and integration, not only in the field of computational techniques, but also at the level of the software code itself. This challenging task has been tackled and the first crucial steps have been set. Basic tools and procedures needed for such cooperation have been defined and developed. Of course, the work is not finished. We still have a long way to go ... Our personal hope, as leaders of this ACE activity, is that the change in mentality that we have seen growing among the partners, clearly going in a direction towards much more intensive cooperation does not fade away after ACE ...

\section{References}

[1] G. A. E. Vandenbosch and R. Gillard, "Goals and structuring of the Antenna Software Initiative (ASI) within ACE", Proc. of the European Conference on Antennas and Propagation 2006, Nice, France, Nov. 2006.

[2] www.antennasvce.org 